\title{
DC Current and Torque Ripple Mitigation in Modular PMSG Drives for Multi-MW WECSs with Linear PWM Inverter Modulation
}

\author{
A. Di Gerlando, K. ElShawarby, G.M. Foglia, M.F. Iacchetti, R. Perini
}

\begin{abstract}
The paper analyzes a torque and DC-current ripple mitigation strategy in a modular axial-flux permanent magnet synchronous generator for multi-MW Wind Energy Conversion Systems (WECSs). The machine modules are aligned with one another and driven by the same shaft. Each module is connected to its own two-level converter and the converters are paralleled at the DC side. In order to reduce the DC-side current harmonics and the ripple in the resultant torque, a phase shift between the converters' PWM carrier signals is implemented. The converters are operated within the linear PWM modulation range. A mathematical analysis is carried out. A criterion for the best choice of the phase shift in two- and three-module configurations is developed. Theoretical results are compared with those from simulations in Matlab/Simulink, verifying the soundness of the method.
\end{abstract}

Index Terms - Multi Modular converters, multi-modular electrical machines, de current ripple mitigation, converter sequential command

\section{INTRODUCTION}

Nowadays, direct-drive systems with Permanent Magnet Synchronous Generators (PMSG) are widely used, because of the high torque density and efficiency, and the absence of gearbox [1], [2].

The connection of wind generators to the grid is usually through a two-stage conversion comprising a DC-bus. For generators in the multi-MW range, multilevel inverters (MLIs) are a viable solution to reduce current and torque ripples with acceptable switching frequencies [3]-[4]. A comprehensive study of MLIs with different topologies used for renewable energy applications is mentioned in [5]. Compared to pulse-width modulation (PWM) driven twolevel converters, MLI-drives in general have lower harmonic injection and lesser torque ripple for the same switching frequency [6]-[7]. However, they have a higher number of switching devices that increase costs. Moreover, these switches need related gate circuitry that increases the overall system complexity [8]-[9].

A. Di Gerlando, K. ElShawarby, G.M. Foglia, R. Perini are with the Dpt. of Energy at Politecnico di Milano, Via La Masa 34, 20156 Milano - Italy e-mails: antonino.digerlando@polimi.it, khaled.elshawarby@polimi.it, gianmaria.foglia@polimi.it, roberto.perini@polimi.it

M.F. Iacchetti is with School of Electrical and Electronic Engineering, University of Manchester (UK); e-mail: matteo.iacchetti@manchester.ac.uk
This paper analyses a sequential command technique for the converters for a modular axial-flux PMSGs used in wind energy applications. In the considered modular PMSG, each generator module is connected to a dedicated two-level converter. All these converters can be connected in parallel.

The high degree of electromechanical and converter modularity of this kind of system ensures the following advantages:

- the use of two-level standard converters based on a very mature technology;

- the reduction of voltage and current ratings of each module, making it possible to use standard off-the-shelf power electronics;

- a more effective reliability and redundancy, thanks to the absence of magnetic mutual coupling between modules;

- the mitigation of ripples in torque and DC-link current through a coordinated control of the converters.

- the parallel connection does not cause the current circulation across the converters, discussed in [10], since the AC side of each converter is connected to a separate three-phase machine module.

The generator-side converters are operated with suited sequential shift of the carrier signals in order to improve the waveforms of DC-side current and electromagnetic torque. A similar approach was adopted in [11] investigating the same machine. However, in [11] the stators of the machine were reciprocally phase shifted by a given angle, instead of being mechanically aligned. Moreover, the modulation was limited to square wave modulation. However, in this paper, all machine modules are aligned with each other and the modulation technique and the analysis of DC-side current and torque is limited to PWM modulation within the linear range. A criterion is developed to show the exact shift angle in the triangular carrier signals of the AC-DC converters in order to reduce the Total Harmonic Distortion (THD) of the global DC current and subsequently decrease the overall torque ripple without affecting the average torque.

The paper is divided as follows: Section II presents the system under study and the rated values of the modular axial flux permanent magnet (AFPM) generator. Section III derives the expression of the instantaneous DC side current in one module with PWM modulation and the phase relationships between ripple harmonics and PWM carrier signal. An instantaneous expression of the electromagnetic torque is also developed. Section IV describes the ripple compensation technique based on the sequential phase shift of carrier signals in neighbouring converters. In Section V the 
comparison between the analytical model and the simulation results is performed. In Section VI a discussion of simulation results is shown; a comparison of the harmonic distortion of the torque for one-, two- and three-module machine at different switching frequency of the AC-DC converters is performed.

\section{STRUCTURE AND CONTROL}

The modular AFPM synchronous generator under study is represented in Fig.1a: all the $N_{m}$ rotors are fastened on the same shaft and aligned with each other, stator windings across the machine are aligned as well. Each module is connected to a two-level AC-DC converter and all the converters are paralleled at the DC side; $i_{d c \mu}$ is the DC-current of module $\mu\left(\mu=1,2 \ldots N_{m}\right), i_{d c t}$ is the resultant DC current.

The assumed rated data of each module are in Table I.

The low rated speed is consistent with a direct-drive turbinegenerator system. The rating of each module is compatible with the use of two-level converters.

The d-and q-axis inductances have the same value because the machine is isotropic. In addition, the sub-transient inductance is close to the synchronous one, since the damping cage is absent and other damping effects are negligible.

TABLE I

RATED DATA OF EACH AFPM MACHINE MODULE.

\begin{tabular}{c|c}
\hline Rated $P_{n}[\mathrm{~kW}], I_{n}[\mathrm{~A}], N_{n}[\mathrm{rpm}]$ & $1000 ; 657 ; 17$ \\
\hline $\begin{array}{c}\text { Line to neutral EMF at rated speed } E_{0}\left[\mathrm{~V}_{R M S}\right] \\
(\text { sinusoidal waveform) }\end{array}$ & 507.5 \\
\hline No of poles $p$, rated frequency $f_{n}[\mathrm{~Hz}]$ & $104 ; 14.73$ \\
\hline Stator flux linkage due to PM, $\Psi_{P M}=E_{0} /\left(2 \pi f_{n}\right)[\mathrm{Wb}]$ & 5.48 \\
\hline Phase resistance $R[\mathrm{~m} \Omega]$ & 16.15 \\
\hline Synchronous inductance $(\mathrm{d}, \mathrm{q}$ axes $) L[\mathrm{mH}]$ & 3.132 \\
\hline Axial length (one module) $[\mathrm{m}]$ & 0.336 \\
\hline External diameter $[\mathrm{m}]$ & 5.000 \\
\hline Rotor inertia $\left[\mathrm{kg} \mathrm{m}{ }^{2}\right]$ & $3.707 \cdot 10^{4}$ \\
\hline
\end{tabular}

\section{InSTANTANEOUS DC SidE CURRENT IN ONE MOdUlE}

\section{A. General Aspects.}

In this section, the mathematical expression of the current on the DC side will be developed. Thus it will be possible to identify and apply the necessary shift between the triangular carrier signals of neighbouring converters (Fig.1b), in order to decrease the harmonic distortion in the DC side current. The control signals are the same for all the converters.

Reference is made to one single module $\mu$ (in the variables, subscript $\mu$ is discharged, for simplicity). Its equivalent circuit is presented in Fig. 1c: each machine phase is modelled as a series $R-L-e$ circuit. The two-level converter is connected to the DC bus capacitor of voltage $V_{d}$. Point $\mathrm{z}$ represents the virtual midpoint of the DC side capacitor.

Equation (1) reports the analytical harmonic solution for the double-edge naturally-sampled PWM of the three phase converter [12] with sinusoidal modulation.

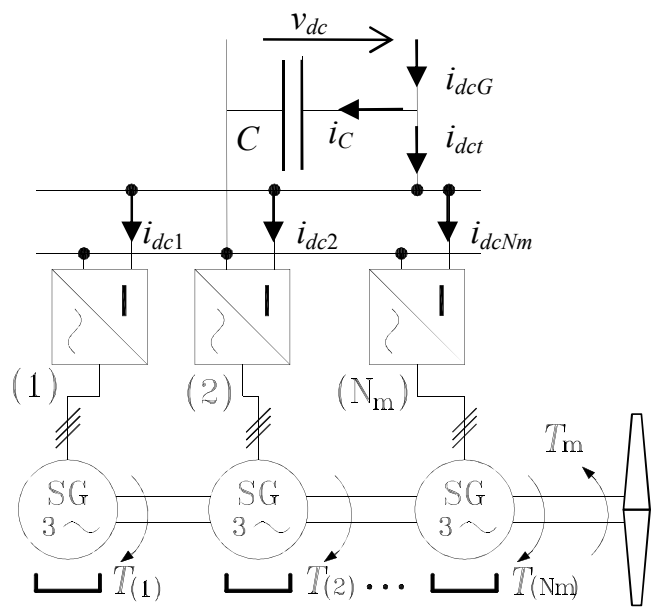

(a)

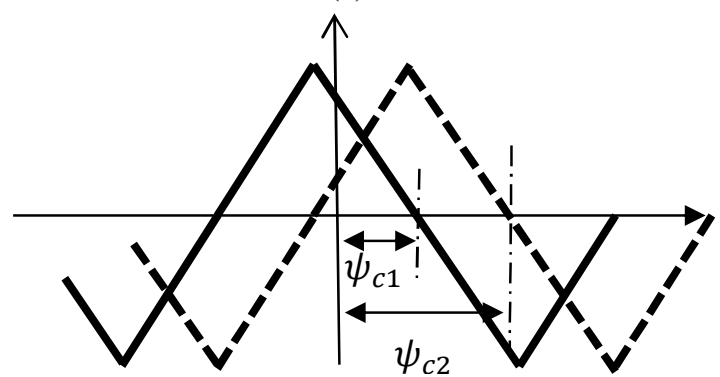

(b)

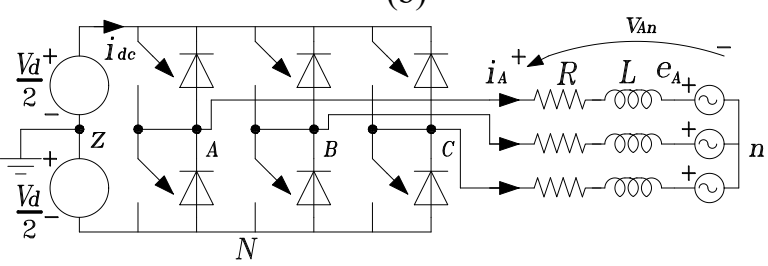

(c)

Fig.1. Machine and power electronics arrangements:

a) Schematic of the modular direct-drive axial-flux PMSG under study. Each module of the machine is connected to a two-level converter. All the twolevel converters are paralleled at the DC side.

b) Proposed shift $\left(\psi_{c 2}-\psi_{c 1}\right)$ introduced in the triangular carrier signal for the converters.

c) Equivalent circuit for one module of the AFPM machine and its converter. The load sign convention is used.

Expression (1) is the voltage between phase A and the midpoint $\mathrm{z}$ of the capacitor; $M$ is the amplitude modulation index of the two level converter; $m_{f}$ is the frequency modulation ratio (as known, usually odd and multiple of 3); $J_{u}$ is the Bessel function of the first kind and order $u ; m$ is the carrier index and $n$ is the base band index for the $h$ harmonic component, due to the PWM modulation: $h=m \cdot m_{f}+n ; \omega_{o}$ is the fundamental angular frequency; $\omega_{c}$ is the carrier angular frequency: $\omega_{c}=m_{f} \cdot \omega_{o}$.

$$
\begin{gathered}
v_{A z}=\frac{V_{d}}{2} M \cos \left(\omega_{o} t\right)+\frac{2 V_{d}}{\pi} \sum_{m=1}^{\infty} \sum_{n=-\infty}^{\infty} \frac{1}{m} J_{u}\left(m \frac{\pi}{2} M\right) \sin \left([m+n] \frac{\pi}{2}\right) \cos \left(m\left[\omega_{c} t+\psi_{c}\right]+n \omega_{o} t\right) \\
v_{B z}=\frac{V_{d}}{2} M \cos \left(\omega_{o} t-\frac{2 \pi}{3}\right)+\frac{2 V_{d}}{\pi} \sum_{m=1}^{\infty} \sum_{n=-\infty}^{\infty} \frac{1}{m} J_{u}\left(m \frac{\pi}{2} M\right) \sin \left([m+n] \frac{\pi}{2}\right) \cos \left(m\left[\omega_{c} t+\psi_{c}\right]+n\left[\omega_{o} t-\frac{2 \pi}{3}\right]\right) \\
v_{C z}=\frac{V_{d}}{2} M \cos \left(\omega_{o} t-\frac{4 \pi}{3}\right)+\frac{2 V_{d}}{\pi} \sum_{m=1}^{\infty} \sum_{n=-\infty}^{\infty} \frac{1}{m} J_{u}\left(m \frac{\pi}{2} M\right) \sin \left([m+n] \frac{\pi}{2}\right) \cos \left(m\left[\omega_{c} t+\psi_{c}\right]+n\left[\omega_{o} t-\frac{4 \pi}{3}\right]\right)
\end{gathered}
$$


The angle $\psi_{c}$ is the phase of the carrier signal applied to a generic converter (Fig. 1b).

\section{B. PWM Harmonic Voltage and Current Amplitude}

The Bessel function $J_{u}$ is simplified to the first three terms of the Taylor series: this is a suited approximation for $M \leq 1$.

Considering only the harmonics that correspond to $m=1$, 2 and $n=0, \pm 1, \pm 2, \pm 3$, the AC voltage amplitudes of the harmonics can be calculated, as a function of $M$, Table II, where the harmonic orders derive from $h=m \cdot m_{f}+n$.

Called $L, R$ the machine equivalent inductance and resistance respectively, the $h$-th harmonic equivalent impedance amplitude and characteristic angle are:

$$
\begin{gathered}
Z_{h}=\sqrt{R^{2}+\left(h \omega_{o} L\right)^{2}} \\
\theta_{h}=\tan ^{-1}\left(\frac{h \omega_{o} L}{R}\right) .
\end{gathered}
$$

Called $V_{h}$ the RMS values of the $\mathrm{AC}$ voltage harmonic, the RMS values of the AC current harmonic is:

$$
I_{h}=\frac{V_{h}}{Z_{h}} \quad h>1
$$

TABLE II

HaRmONIC RMS Values OF THE AC Voltage $v_{a z}$ (Evaluated By (1), WITH $m=1,2 ; n=0, \pm 1, \pm 2, \pm 3$ ONLY).

$$
\begin{gathered}
V_{1}=\frac{V_{d}}{2 \sqrt{2}} \cdot M \\
V_{m_{f}}=\frac{\sqrt{2} V_{d}}{\pi} \cdot\left(1-\frac{\pi^{2} M^{2}}{16}+\frac{\pi^{4} M^{4}}{1024}\right) \\
V_{m_{f} \mp 2}=\frac{-\sqrt{2} V_{d}}{\pi} \cdot \frac{(\pi M)^{2}}{32}\left(1-\frac{\pi^{2} M^{2}}{48}+\frac{\pi^{4} M^{4}}{6144}\right) \\
V_{2 m_{f} \mp 1}=-\frac{\sqrt{2} V_{d}}{\pi} \cdot \frac{\pi M}{4}\left(1-\frac{\pi^{2} M^{2}}{8}+\frac{\pi^{4} M^{4}}{192}\right) \\
V_{2 m_{f} \mp 3}=\frac{\sqrt{2} V_{d}}{\pi} \cdot \frac{(\pi M)^{3}}{96}\left(1-\frac{\pi^{2} M^{2}}{16}+\frac{\pi^{4} M^{4}}{640}\right)
\end{gathered}
$$

C. PWM Harmonic Voltage and Current Expressions on the AC Side

As for phase A, the expression of the voltage after applying the shift in the carrier signal and considering the chosen harmonics is:

$$
\begin{aligned}
v_{A z} & =\sqrt{2} V_{1} \cos \left(\omega_{o} t\right)+\sum_{n=-2,0,2} \sqrt{2} V_{m_{f}+n} \cos \left(\omega_{c} t+\psi_{c}+n \omega_{o} t\right)+ \\
& +\sum_{n=-3,-1,1,3} \sqrt{2} V_{m_{f}+n} \cos \left(\omega_{c} t+\psi_{c}+n \omega_{o} t\right)
\end{aligned}
$$

As for phases B and C, a phase shift of $n \cdot 120$ degrees or $n \cdot 240$ degrees is applied, respectively: this is the only difference among the equations.

In order to find the current flowing through the module, the machine phase voltages with respect to the neutral point $n$ are calculated, by means of the known relations:

$$
\left\{\begin{array}{l}
v_{A n}(t)=v_{A z}-\frac{1}{3} \cdot\left(v_{A z}+v_{B z}+v_{C Z}\right) \\
v_{B n}(t)=v_{B z}-\frac{1}{3} \cdot\left(v_{A z}+v_{B z}+v_{C Z}\right) \\
v_{C n}(t)=v_{C z}-\frac{1}{3} \cdot\left(v_{A z}+v_{B z}+v_{C Z}\right)
\end{array}\right.
$$

If $m_{f}$ is multiple of 3 , some voltage harmonics will be cancelled out, namely $v_{m f}, v_{2 m f-3}, v_{2 m f+3}$, due to neutral point. That means that the corresponding current harmonics $i_{m f}$, $i_{2 m f-3}, i_{2 m f+3}$ will not exist.

The current harmonic equations will have the same structure of the voltage but with a shift in the angle, due to the corresponding impedance:

$$
i_{h}(t)=\sqrt{2} I_{h} \cos \left(h \omega_{o} t+\angle \bar{V}_{h}-\theta_{h}\right)
$$

The voltage phase $\angle \overline{\mathrm{V}}_{\mathrm{h}}$ includes the dependence on the phase angle $\psi_{\mathrm{c}}$ of the carrier signal.

For each module, the phase currents $i_{A}, i_{B}, i_{C}$, are obtained by summing the several current harmonics.

\section{Instantaneous DC Current Ripple}

Assuming a constant DC voltage $V_{d}$ and lossless converter, the power balance across the converter of one module yields:

$$
V_{d} \cdot i_{d c}(t)=i_{A}(t) \cdot v_{A n}(t)+i_{B}(t) \cdot v_{B n}(t)+i_{C}(t) \cdot v_{C n}(t)(8)
$$

Products on the right-hand side of (8) give two effects. The interaction between currents and voltages of the same harmonic order $i_{x} \cdot v_{x}$ will give a contribution to the average value $I_{d c}$ of the current on the DC side. The interaction between currents and voltages of different harmonic orders $i_{x} \cdot v_{y}(x \neq y)$ produces the ripple component $i_{r}$.

As an example, the expression for the ripple component is now derived considering the interaction between the fundamental voltage and the current of harmonic order $m_{f}-2$ :

$$
\begin{gathered}
\frac{v_{A n_{1}} i_{A m f-2}}{V_{d}}=\frac{\sqrt{2} V_{1}}{V_{d}} \cos \left(\omega_{o} t\right) . \\
\cdot\left[\sqrt{2} I_{m f-2} \cos \left(\omega_{c} t+\psi_{c}-2 \omega_{o} t-\theta_{m f-2}\right)\right]
\end{gathered}
$$

The expression can be expanded as follows:

$$
\begin{gathered}
\frac{v_{A n_{1}} i_{A m f-2}}{V_{d}}=\frac{V_{1} I_{m f-2}}{V_{d}} \cdot\left[\operatorname { c o s } \left(\omega_{c} t+\psi_{c}-\omega_{\mathrm{o}} t-\right.\right. \\
\left.\left.\theta_{m f-2}\right)+\cos \left(\omega_{\mathrm{c}} t+\psi_{c}-3 \omega_{\mathrm{o}} t-\theta_{m f-2}\right)\right]
\end{gathered}
$$

And similarly for phases B and C,

$$
\begin{aligned}
& \frac{v_{B n_{1}} i_{B m f-2}}{V_{d}}=\frac{V_{1} I_{m f-2}}{V_{d}} \cdot\left[\operatorname { c o s } \left(\omega_{c} t+\psi_{c}-\omega_{\mathrm{o}} t-\theta_{m f-2}+\right.\right. \\
& \left.\left.120^{\circ}\right)+\cos \left(\omega_{c} t+\psi_{c}-3 \omega_{\mathrm{o}} t-\theta_{m f-2}\right)\right] \\
& \frac{v_{C n_{1}} i_{C_{m f-2}}}{V_{d}}=\frac{V_{1} I_{m f-2}}{V_{d}} \cdot\left[\operatorname { c o s } \left(\omega_{c} t+\psi_{c}-\omega_{\mathrm{o}} t-\theta_{m f-2}-\right.\right. \\
& \left.\left.120^{\circ}\right)+\cos \left(\omega_{\mathrm{c}} t+\psi_{c}-3 \omega_{\mathrm{o}} t-\theta_{m f-2}\right)\right]
\end{aligned}
$$

Recalling $\omega_{c}=m_{f} \cdot \omega_{0}$, two harmonic components of order $m_{f}-1$ and $m_{f}-3$ coexist. As for the former, it is always zero, because the components of the three phases are displaced by 120 degrees:

$$
i_{r, m f-1}(t)=0
$$

As for the latter, it follows:

$i_{r, m f-3}(t)=\frac{3 V_{1} I_{m f-2}}{V_{d}} \cos \left(\omega_{c} t+\psi_{c}-3 \omega_{o} t-\theta_{m f-2}\right)$

Due to the absence of any shift between the three phases, 
the summation is not equal to zero. In fact, it is three times the contribution of one phase; therefore, it will add to the expression of the DC side current ripple.

The complete expression of $i_{r \mu}(\mathrm{t})$ is reported in (A1) in the Appendix. The values of $h$ and the phase shift of the carrier signal are reported in Table III.

TABLE III

HARMONIC CONTENT IN THE DC-BUS CURRENT AND ELECTROMAGNETIC TORQUE, AND PHASE OF EACH HARMONIC

\begin{tabular}{c|c}
\hline $\boldsymbol{h}$ & phase of each harmonic \\
\hline $\boldsymbol{m}_{\boldsymbol{f}} \pm \mathbf{3}$ & $\psi_{c}$ \\
\hline $\mathbf{2} \boldsymbol{m}_{\boldsymbol{f}}$ & $2 \psi_{c}$ \\
\hline $\mathbf{3} \boldsymbol{m}_{\boldsymbol{f}} \pm \mathbf{3}$ & $3 \psi_{c}$ \\
\hline $\mathbf{4} \boldsymbol{m}_{\boldsymbol{f}}$ & $4 \psi_{c}$ \\
\hline
\end{tabular}

\section{E. Instantaneous Torque Ripple of One Module.}

Each module is vector-controlled to operate in maximum torque-per-ampere conditions. By adopting the motoring sign convention, the angle between first harmonics of current $\bar{I}_{1}$ and internal EMF $\bar{E}$ is 180 degrees (Fig. 2).

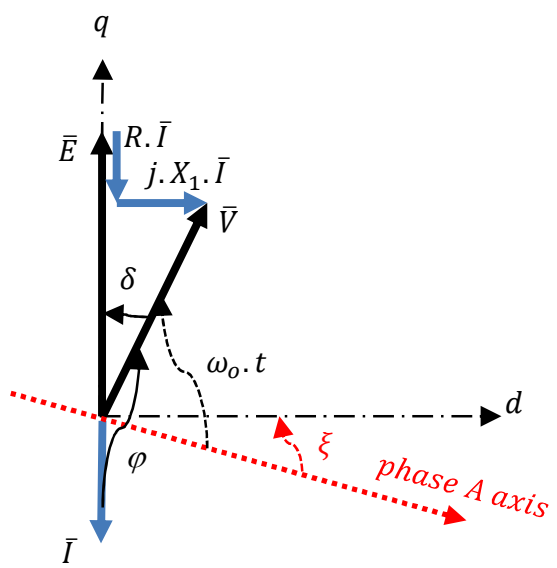

Fig. 2. Phasor diagram of the first harmonic component of one module, the internal EMF $\bar{E}$ is shifted by 180 degrees to obtain maximum torque.

Using the dq axis theory, with the d-axis aligned to the PM flux $\psi_{P M}$ of the module, the expression of the electromagnetic torque given by each $\mu_{t h}$ module is (see Table I):

$$
T_{e l m \mu}=(p / 2) \sqrt{3} \Psi_{P M} i_{q \mu},
$$

where the q-axis current component $i_{q \mu}$ of the $\mu_{t h}$ module is:

$$
\begin{gathered}
i_{q \mu}=-\sqrt{2 / 3} \cdot\left[\sin (\xi) \cdot i_{A \mu}+\sin (\xi-2 \pi / 3) \cdot i_{B \mu}\right. \\
\left.+\sin (\xi-4 \pi / 3) \cdot i_{C \mu}\right]
\end{gathered}
$$

and the electrical angle $\xi$ between $\mathrm{d}$ axis and phase $\mathrm{A}$ axis equals (see Fig. 2):

$$
\xi=\omega_{0} t+\delta-\pi / 2
$$

In modular AFPM machines, the phase current systems $\left\{i_{A}, i_{B}, i_{C}\right\}$ of the several modules have their ripple components that are shifted, according to the theory developed previously. It can be noted that the dc components of $\mathrm{d}-\mathrm{q}$ frame for all modules are overlapping while each group of harmonics has a distinctive phase shift multiple of $\psi_{c}$ as shown in Table III.

Then, the machine global electromagnetic torque can be found by summing all the contributions:

$$
T_{\text {elmt }}(t)=\sum_{\mu=1}^{N m} T_{\text {elm } \mu}(t)
$$

\section{HARMONIC ELIMINATION IN THE DC CURRENT}

By summing the expressions of the several $i_{r \mu}(t)$, the expression of the resultant DC current ripple $i_{r t}(t)$ is obtained. Thus, it can be possible to select any specific harmonic and eliminate it by selecting the proper phase shift of the carrier among converters. In the following the harmonic of order $\left(m_{f}-3\right)$ is chosen to be eliminated.

\section{A. Adoption of $N_{m}=2$ Converter Modules.}

Let us consider the case when the machine has two modules where each module is connected to a converter. $\psi_{\mathrm{cl}}$ and $\psi_{c 2}$ are the phases of the carrier signals of the two converters respectively. In order to eliminate the resultant harmonic of order $\left(m_{f}-3\right)$, the phase displacement between the two carrier signals must be equal to:

$$
\Delta \psi_{c \text { two-mod }}=\psi_{c 2}-\psi_{c 1}=180 \mathrm{deg}
$$

Additionally, this choice will also eliminate the harmonics of order $\left(m_{f}+3\right),\left(3 \cdot m_{f}-3\right),\left(3 \cdot m_{f}+3\right)$. Therefore, the expression of the resultant DC current ripple $i_{r t}(t)_{\text {two-mod }}$ has only $\left(2 \cdot m_{f}\right),\left(4 \cdot m_{f}\right)$ components which will add as shown in (A2) in the Appendix.

\section{B. Adoption of $N_{m}=3$ Converter Modules.}

In order to eliminate the resultant harmonic $\left(m_{f}-3\right)$ using three modules, the phase displacement between the three carrier signals must equal:

$$
\Delta \psi_{c \text { three-mod }}=\psi_{c 2}-\psi_{c 1}=\psi_{c 3}-\psi_{c 2}=120 \mathrm{deg}
$$

This solution will also eliminate the harmonics $\left(m_{f}+3\right)$, $\left(2 m_{f}\right),\left(4 m_{f}\right)$. Therefore the expression of the resultant DC current ripple $i_{r t}(t)_{\text {three-mod }}$ has only the harmonic $\left(3 m_{f} \pm 3\right)$. The expression can be obtained in the same manner as the two-module one (see the Appendix).

\section{COMPARISON BETWEEN SIMULATION RESULTS AND THEORETICAL MODEL}

The rated conditions for one module can be calculated (see Fig. 2 for the phasor diagram):

$$
T_{n}=T_{\text {elm } n} ; I_{d}=0 ; I_{q}=\sqrt{ } 3 I_{n} ; \delta=22.81 \mathrm{deg} ; V_{\text {phase }}=526 \mathrm{~V}_{\mathrm{RMS}}
$$

The results of the theoretical analysis are reported in Table IV and $\mathrm{V}$, as for the resultant DC current and torque, for the cases with $N_{m}=1,2$ and 3 modules. The comparison has been carried out with $m_{f}=15$ (carrier frequency $f_{c}=221 \mathrm{~Hz}$ ).

These results are compared with those of a Matlab/Simulink simulation with the same rated operating conditions. Each machine module is modelled by the standard two-axis (dq) model of the PM brushless machine. For each module, the classical field oriented control is implemented, aimed at maximizing the torque-per-ampere ratio (see Fig. 2). Two cascaded controllers are implemented: current (internal) and speed (external) controllers; their band-passes equal 100 
$\mathrm{rad} / \mathrm{s}$ and $10 \mathrm{rad} / \mathrm{s}$. The carrier signals are shifted with respect to each other according to the shift value obtained in Sect. IV.

By extending the number of terms in (1)-(2) (so that $m \leq 3$ and $|n| \leq 4)$, good accuracy can be obtained up to $60^{\text {th }}$ harmonic as shown in Tables IV and V.

Two matters are apparent from Tables IV and V:

1) dc side current and electromagnetic torque have the same harmonic contents;

2) pu torque ripple is much lower than DC current one (see Fig. 3, which shows the p.u. torque and current ripple for one module).

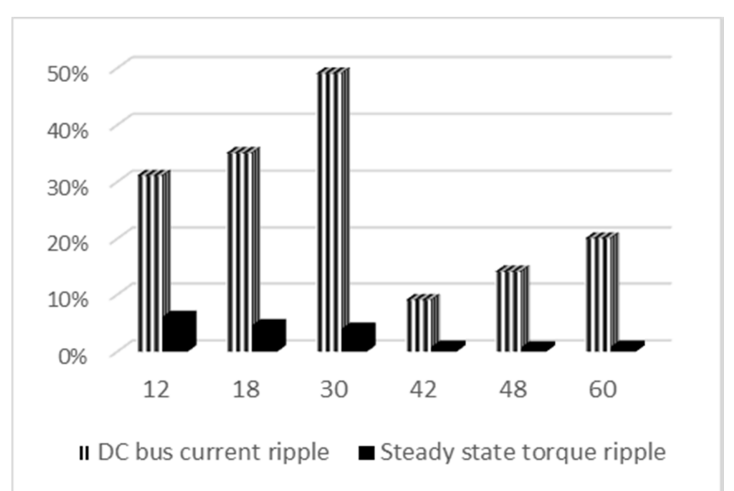

Fig. 3. Comparison between the harmonic p.u. amplitudes of both current and torque studied for switching frequency ratio $m_{f}=15$, considering only one module of the machine, operating in rated conditions.

\section{TABLE IV}

THEORETICAL AND SIMULATED RESULTS DC CURRENT HARMONIC COMPONENTS REFERRED TO $I_{d c}$ $m=1,2,3 ; n=0, \pm 1, \pm 2, \pm 3, \pm 4$ AT $m_{f}=15$

\begin{tabular}{l|c|c|c|c|c|c}
\hline Modules & \multicolumn{2}{|c|}{$N_{m}=1$} & \multicolumn{2}{c|}{$N_{m}=2$} & \multicolumn{2}{c}{$N_{m}=3$} \\
\hline & theor. & sim. & theor. & sim. & theor. & sim. \\
\hline$I_{d c}[\mathrm{~A}]$ & 613 & 610 & 1225 & 1219 & 1838 & 1829 \\
\hline$I_{12}[\%]$ & 28.3 & 31.5 & 0 & 0 & 0 & 0 \\
\hline$I_{18}[\%]$ & 34.6 & 34.5 & 0 & 0 & 0 & 0 \\
\hline$I_{30}[\%]$ & 49.3 & 51.2 & 49.4 & 51.0 & 0 & 0 \\
\hline$I_{42}[\%]$ & 8.14 & 8.77 & 0 & 0 & 8.13 & 9.37 \\
\hline$I_{48}[\%]$ & 13.6 & 12.8 & 0 & 0 & 13.6 & 13.3 \\
\hline$I_{60}[\%]$ & 19.5 & 19.7 & 19.4 & 20.6 & 0 & 0 \\
\hline
\end{tabular}

TABLE V

THEORETICAL AND SIMULATED RESULTS TORQUE HARMONIC COMPONENTS REFERRED TO $T_{d c}$ $m=1,2,3 ; n=0, \pm 1, \pm 2, \pm 3, \pm 4$ AT $m_{f}=15$

\begin{tabular}{l|c|c|c|c|c|c}
\hline Modules & \multicolumn{2}{|c|}{$N_{m}=1$} & \multicolumn{2}{c|}{$N_{m}=2$} & \multicolumn{2}{c}{$N_{m}=3$} \\
\hline & theor. & sim. & theor. & sim. & theor. & sim. \\
\hline$T_{d c}[\mathrm{kNm}]$ & 562 & 560 & 1124 & 1119 & 1686 & 1679 \\
\hline$T_{12}[\%]$ & 5.20 & 6.10 & 0 & 0 & 0 & 0 \\
\hline$T_{18}[\%]$ & 4.34 & 4.60 & 0 & 0 & 0 & 0 \\
\hline$T_{30}[\%]$ & 3.91 & 4.00 & 3.91 & 4.0 & 0 & 0 \\
\hline$T_{42}[\%]$ & 0.56 & 0.80 & 0 & 0 & 0.56 & 0.70 \\
\hline$T_{48}[\%]$ & 0.58 & 0.70 & 0 & 0 & 0.58 & 0.59 \\
\hline$T_{60}[\%]$ & 0.77 & 0.90 & 0.77 & 0.90 & 0 & 0 \\
\hline
\end{tabular}

Both matters are explained by the power balance. The DC power $p_{d c}$ is subdivided into the losses $p_{\text {loss }}$, the rate of change of energy stored in the machine inductance $p_{\text {induc }}$, the mechanical power $p_{\text {mech }}$.

$$
p_{d c}=p_{\text {loss }}+p_{\text {induc }}+p_{\text {mech }} .
$$

By expanding the terms, the expression becomes:

$$
V_{d} i_{d c}=p_{\text {loss }}+\frac{1}{2} L \frac{d}{d t}\left(i_{d}^{2}+i_{q}^{2}\right)+T_{e l m} \Omega .
$$

By neglecting the losses, the torque is approximated to:

$$
T_{\text {elm }} \approx \frac{1}{\Omega}\left[p_{d c}-p_{\text {induc }}\right]=\frac{V_{d}}{\Omega}\left[i_{d c}-\frac{1}{2} \frac{L}{V_{d}} \frac{d}{d t}\left(i_{d}^{2}+i_{q}^{2}\right)\right]
$$

Eq. (22) shows the direct correlation between the dc side current and the electromagnetic torque, explaining why the existing harmonic orders are the same. Moreover, it is also apparent that most of the DC instantaneous power due to the current ripple is absorbed by the machine inductance, while only a small portion contributes to the electromagnetic torque: thus, torque ripple results are much lower than DC current one.

\section{SimUlation WAVEFORMS AND DiSCUSSION}

To better appreciate the soundness of the proposed method, some waveforms obtained by Matlab/Simulink simulations are reported in the following.

Using the equation (A1) in Appendix, we can calculate all the harmonic amplitudes at all speeds.

Fig.4 shows a comparison between the torque THD for a one-module, two-module (carriers shifted by $180 \mathrm{deg}$. among them) and three-module machine (carriers shifted by 120 and 240 deg. among them). As shown, the shifted three-module system has much lower torque THD than the original one.

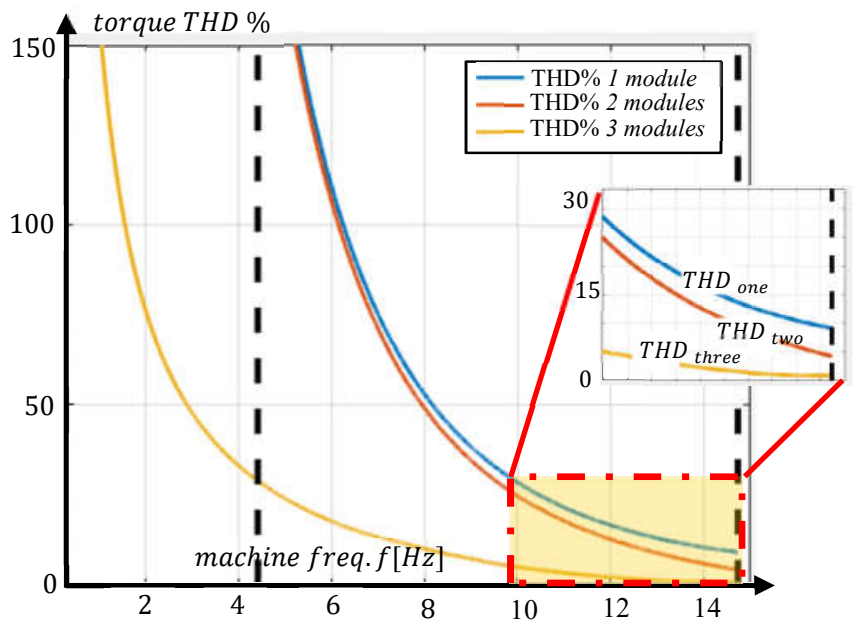

Fig. 4. Comparison between torque THD, across the whole range of operation $\left(0.3 \sim 1\right.$ of the rated frequency), at a frequency ratio $m_{f}=15$, for one module, two modules with shift of $180^{\circ}$ and three modules with shift of $120^{\circ}, 240^{\circ}$. Vertical dashed lines are the cut-in and cut-out frequencies.

Fig. 5a shows the electromagnetic torque and the dc side current with the one-module configuration.

Fig. 6a presents the electromagnetic torque due to each module in a three-module configuration and the resultant torque; the waveforms are shifted among one another ; along 
the vertical axis, on the left, the average value is reported, on the right, the peak-to-peak variation is shown. It can be seen that the torques of each module are phase shifted with respect to each other according to the criterion developed in Section IV and their sum is characterised by a much lower ripple.
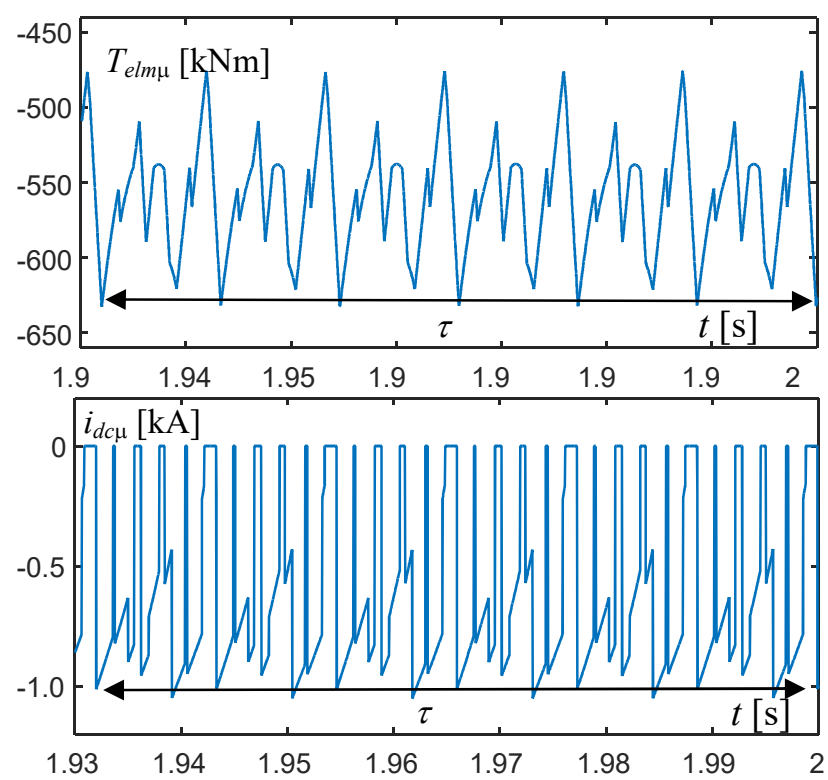

Fig. 5. Waveforms in the one-module configuration ( $\tau$ : fundamental period). a) Electromagnetic torque: notice the wide harmonic component of order $\left(m_{f}-3=12\right)$. b) DC-bus current:
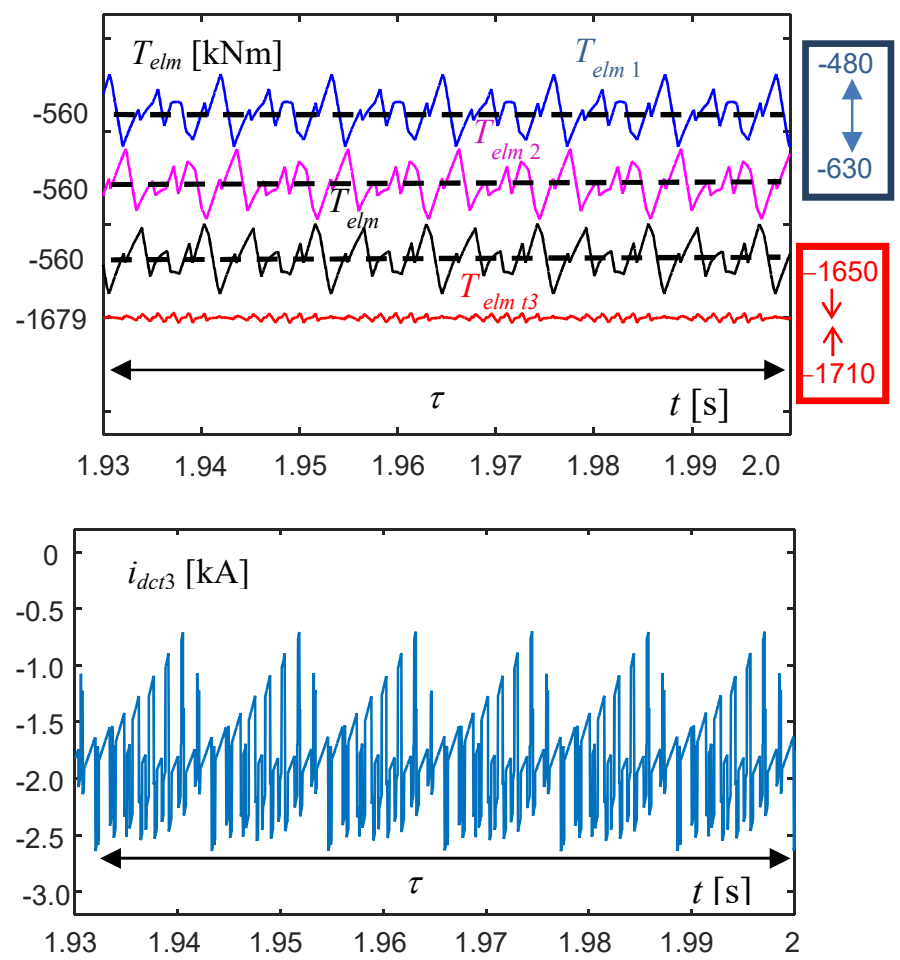

Fig. 6. Waveforms in the three-module configuration ( $\tau$ : fundamental period). a: electromagnetic torque. b: DC-bus current.

Figs. $5 b, 6 b$ report the DC current waveform for the one-, three-module configurations, respectively.

Comparing Fig. 6b with Fig. 5b, it follows:

- the pu peak-to-peak ripple (referred to its own average DC current) has considerably decreased;

- time intervals with zero current due to the free-wheeling diode conduction disappear in resultant current

There is another advantage of the sequential control of parallel converter: it allows using lower switching frequencies keeping limited ripple of both the DC current and the torque. Table VI reports the THD of the resultant torque in one-, two- and three-module machines, at different frequency modulation ratios. As $m_{f}$ (hence the switching frequency) increases, the torque THD decreases, but the switching losses in the converters rise. On the other side, keeping $m_{f}$ constant and increasing the number of paralleled modules with sequential control reduces the torque THD.

As an example, bold values in Table VI show that a frequency modulation ratio $m_{f}=27$ with only one converter gives a torque $\mathrm{THD}=4.72 \%$; a similar value $(\mathrm{THD}=4.12 \%)$ is obtained by $m_{f}=15$ with two modules connected in parallel and their carrier signals shifted from each other by 180 degrees.

TABLE VI.

TORQUE THD FOR ONE-, TWO- AND THREE-MODULE MACHINE $\left(N_{m}\right)$ AT DIFFERENT FREQUENCY MODULATION RATIOS $\left(m_{f}\right)$.

\begin{tabular}{lrrr}
\hline & $N_{m}=1$ & $N_{m}=2$ & $N_{m}=3$ \\
\hline$m_{f}=15$ & $8.79 \%$ & $\mathbf{4 . 1 2} \%$ & $1.05 \%$ \\
$m_{f}=27$ & $\mathbf{4 . 7 2} \%$ & $2.32 \%$ & $0.58 \%$ \\
$m_{f}=69$ & $1.83 \%$ & $0.91 \%$ & $0.23 \%$ \\
\hline
\end{tabular}

\section{CONCLUSION}

The paper analyzed a technique to reduce ripples of DCcurrent and torque in modular axial-flux PMSG drives for wind power generation. In the considered layout, several independent machine modules are driven by a common shaft and properly aligned with one another. The module ratings allow the connection of each module to a dedicated two-level converter. The resulting multi-MW modular drive maximizes redundancy and reliability using off-the-shelf power electronics converters instead of a custom multi-level converter. The reduction of ripples is achieved by a proper phase-shift of the carrier signals of converters, operated in linear PWM modulation range. Approximated expressions for DC-current and torque ripples have been derived and the optimal phase-shift has been identified as a function of the number of modules.

The system has been simulated by Matlab/Simulink that proved the soundness of the proposed technique. A comparison between the results obtained from the mathematical expressions and from the simulations was performed.

A scaled prototype is being prepared, and future activity will concern experimental tests on it.

\section{APPENDIX}

Expression of the DC side current ripple $i_{r \mu}(t)$ of one module (from (8)):

$$
\begin{gathered}
i_{r \mu}(t)=\frac{3 V_{1} I_{m f-2}}{V_{d}} \cos \left(\left(m_{f}-3\right) \omega_{\mathrm{o}} \mathrm{t}+\psi_{c}-\theta_{m f-2}\right) \\
+\frac{3 V_{1} I_{m_{f}+2}}{V_{d}} \cos \left(\left(m_{f}+3\right) \omega_{\mathrm{o}} \mathrm{t}+\psi_{c}-\theta_{m f+2}\right) \\
+\frac{3 V_{1} I_{2 m_{f}-1}}{V_{d}} \cos \left(\left(2 m_{f}\right) \omega_{o} t+2 \psi_{c}-\theta_{2 m f-1}\right)
\end{gathered}
$$




$$
\begin{aligned}
& +\frac{3 V_{1} I_{2 m_{f}+1}}{V_{d}} \cos \left(\left(2 m_{f}\right) \omega_{\mathrm{o}} \mathrm{t}+2 \psi_{c}-\theta_{2 m f+1}\right) \\
& +\frac{3 V_{m_{f}-2} I_{1}}{V_{d}} \cos \left(\left(m_{f}-3\right) \omega_{\mathrm{o}} \mathrm{t}+\psi_{c}+\phi\right) \\
& +\frac{3 V_{m_{f}-2} I_{m_{f}+2}}{V_{d}} \cos \left(\left(2 m_{f}\right) \omega_{\mathrm{o}} \mathrm{t}+2 \psi_{c}-\theta_{m f+2}\right) \\
& +\frac{3 V_{m_{f}-2} I_{2 m_{f}-1}}{V_{d}} \cos \left(\left(3 m_{f}-3\right) \omega_{\mathrm{o}} \mathrm{t}+3 \psi_{c}-\theta_{2 m f-1}\right) \\
& +\frac{3 V_{m_{f-2}} I_{2 m_{f}+1}}{V_{d}} \cos \left(\left(m_{f}+3\right) \omega_{\mathrm{o}} \mathrm{t}+\psi_{c}-\theta_{2 m f+1}\right) \\
& +\frac{3 V_{m_{f}+2} I_{1}}{V_{d}} \cos \left(\left(m_{f}+3\right) \omega_{\mathrm{o}} \mathrm{t}+\psi_{c}-\phi\right) \\
& +\frac{3 V_{m_{f}+2} I_{m_{f}-2}}{V_{d}} \cos \left(\left(2 m_{f}\right) \omega_{\mathrm{o}} \mathrm{t}+2 \psi_{c}-\theta_{m f-2}\right) \\
& +\frac{3 V_{m_{f}+2} I_{2 m_{f-1}}}{V_{d}} \cos \left(\left(m_{f}-3\right) \omega_{\mathrm{o}} \mathrm{t}+\psi_{c}-\theta_{2 m f-1}\right) \\
& +\frac{3 V_{m f+2} I_{2 m_{f}+1}}{V_{d}} \cos \left(\left(3 m_{f}+3\right) \omega_{\mathrm{o}} \mathrm{t}+3 \psi_{c}-\theta_{2 m f+1}\right) \\
& +\frac{3 V_{2 m_{f}-1} I_{1}}{V_{d}} \cos \left(\left(2 m_{f}\right) \omega_{\mathrm{o}} \mathrm{t}+2 \psi_{c}-\phi\right) \\
& +\frac{3 V_{2 m_{f}-1} I_{m_{f}-2}}{V_{d}} \cos \left(\left(3 m_{f}-3\right) \omega_{\mathrm{o}} \mathrm{t}+3 \psi_{c}-\theta_{m f-2}\right) \\
& +\frac{3 V_{2 m_{f-1}} I_{m_{f}+2}}{V_{d}} \cos \left(\left(m_{f}-3\right) \omega_{\mathrm{o}} \mathrm{t}+\psi_{c}+\theta_{m f+2}\right) \\
& +\frac{3 V_{2 m_{f}-1} I_{2 m_{f}+1}}{V_{d}} \cos \left(\left(4 m_{f}\right) \omega_{\mathrm{o}} \mathrm{t}+4 \psi_{c}-\theta_{2 m f+1}\right) \\
& +\frac{3 V_{2 m f+1} I_{1}}{V_{d}} \cos \left(\left(2 m_{f}\right) \omega_{\mathrm{o}} \mathrm{t}+2 \psi_{c}+\phi\right) \\
& +\frac{3 V_{2 m f+1} I_{m f-2}}{V_{d}} \cos \left(\left(m_{f}+3\right) \omega_{\mathrm{o}} \mathrm{t}+\psi_{c}+\theta_{m f-2}\right) \\
& +\frac{3 V_{2 m_{f}+1} I_{m_{f}+2}}{V_{d}} \cos \left(\left(3 m_{f}+3\right) \omega_{\mathrm{o}} \mathrm{t}+3 \psi_{c}-\theta_{m f+2}\right) \\
& +\frac{3 V_{2 m_{f}+1} I_{2 m_{f}-1}}{V_{d}} \cos \left(\left(4 m_{f}\right) \omega_{\mathrm{o}} \mathrm{t}+4 \psi_{c}-\theta_{2 m f-1}\right)
\end{aligned}
$$

Expression of the resultant DC current ripple $i_{r t}(t)_{t w o-m o d}$ for the case $N_{m}=2$ (using a phase shift between the two converters of $180^{\circ}$ between them).

$$
\begin{gathered}
i_{r t}(t)_{t w o-m o d}= \\
=\frac{6 V_{1} I_{2 m_{f}-1}}{V_{d}} \cos \left(\left(2 m_{f}\right) \omega_{\mathrm{o}} \mathrm{t}+2 \psi_{c}-\theta_{2 m f-1}\right) \\
+\frac{6 V_{1} I_{2 m_{f}+1}}{V_{d}} \cos \left(\left(2 m_{f}\right) \omega_{o} t+2 \psi_{c}-\theta_{2 m f+1}\right) \\
+\frac{6 V_{m_{f}-2} I_{m_{f}+2}}{V_{d}} \cos \left(\left(2 m_{f}\right) \omega_{\mathrm{o}} \mathrm{t}+2 \psi_{c}\right. \\
\left.-\theta_{m f+2}\right) \\
+\frac{6 V_{m_{f+2} I_{m_{f}-2}} \cos \left(\left(2 m_{f}\right) \omega_{\mathrm{o}} \mathrm{t}+2 \psi_{c}-\theta_{m f-2}\right)}{V_{d}}+\frac{6 V_{2 m_{f}-1} I_{1}}{V_{d}} \cos \left(\left(2 m_{f}\right) \omega_{o} t+2 \psi_{c}-\phi\right) \\
+\frac{6 V_{2 m_{f}-1} I_{2 m_{f}+1}}{V_{d}} \cos \left(\left(4 m_{f}\right) \omega_{o} t+4 \psi_{c}-\theta_{2 m f+1}\right) \\
+\frac{6 V_{2 m_{f}+1} I_{1}}{V_{d}} \cos \left(\left(2 m_{f}\right) \omega_{o} t+2 \psi_{c}+\phi\right) \\
+\frac{6 V_{2 m_{f}+1} I_{2 m_{f}-1}}{V_{d}} \cos \left(\left(4 m_{f}\right) \omega_{\mathrm{o}} \mathrm{t}+4 \psi_{c}-\theta_{2 m f-1}\right)
\end{gathered}
$$

\section{REFERENCES}

[1] H. Polinder, J. A. Ferreira, B. B. Jensen, A. B. Abrahamsen, K Atallah, R. A. McMahon: 'Trends in Wind Turbine Generator
Systems', IEEE Journ. of Emerging and Selected topics in Power Electronics, 2013, 1, (3), pp. 174-185

[2] R. S. Semken, M. Polikarpova, P. Roytta, J. Alexandrova, J. Pyrhonen, J. Nerg, A. Mikkola,J. Backman: 'Direct-drive permanent magnet generators for high power wind turbines: benefits and limiting factors', IET Renewable Power Generation, 2012, 6, (1), pp. 1-8

[3] X. Yuan, J. Chai, Y. Li: 'A Transformer-Less High-Power Converter for Large Permanent Magnet Wind Generator Systems', IEEE Trans. on Sustainable Energy, 2012, 3, (3), pp. 318-329

[4] X. Yuan:, 'A Set of Multilevel Modular Medium-Voltage High Power Converters for 10-MW Wind Turbines', IEEE Transactions on Sustainable Energy, 2014, 5, (2), pp. 524-534.

[5] Peeyush Kala, Sudha Arora: 'A Comprehensive Study of Classical and Hybrid Multilevel Inverter Topologies for Renewable Energy', Renewable and Sustainable Energy Reviews, 2017, 76, pp. 905-931

[6] G. Grandi, J. Loncarski, O. Dordevic: 'Analysis and Comparison of Peak to Peak Current ripple in two level and multilevel PWM inverters', IEEE Trans on Ind. Electr., 2015, 62, (5), pp. 2721-2730

[7] M. Schweizer, T. Friedli, and J. W. Kolar: 'Comparative Evaluation of Advanced Three-Phase Three-Level Inverter/Converter Topologies Against Two-Level Systems', IEEE Trans. on Ind. Electronics, 2013, 60, (12), pp. 5515-5527

[8] R. Melício, V. M. F. Mendes, J. P. S. Catalão: 'Two-level and Multilevel Converters for Wind Energy Systems: A Comparative Study', Power Electronics and motion control Conf., 2008, pp. 16821687 Poznan

[9] G. I. Orfanoudakis, S. M. Sharkh, M. A. Yuratich, M. A. Abusara: 'Loss comparison of two and three-level inverter topologies', Power Electr., Machines and Drives Conf. (PEMD), 2010 Brighton UK, pp. $1-6$

[10] Chunwei Song, Rongxiang Zhao, Zheng Zeng, Minglei Zhu, Hui Cai: 'Circulating current elimination scheme for parallel operaton of common dc bus inverters', International Journal of Electric Power \& Energy Systems, 2014, 63, pp. 17-29

[11] A. Di Gerlando; K. ElShawarby; G. M. Foglia; R. Perini: 'DC side current and torque ripples reduction in multi modular PMSG for wind applications', XXII International Conference on Electrical Machines (ICEM), 2016, pp. 252-258

[12] D.G. Holmes, B. P. McGrath: 'Opportunities for Harmonic Cancellation with Carrier Based PWM for Two-Level and MultiLevel Cascaded Inverters', Ind. Appl. Conf., Phoenix, AZ (USA),1999, pp. 781-788 Vol.2.

\section{BIOGRAPHIES}

Antonino Di Gerlando received his MS degree in electrical engineering from the Politecnico di Milano, Italy, in 1981. Currently, he is a Full Professor at the Department of Energy at Politecnico di Milano. Fields of interest: design and modeling of electrical machines, converters and drive systems. He is a senior member of IEEE and a member of the Italian Association of the Electric and Electronic Engineers (AEI).

Khaled Elshawarby received his MS degree in electrical engineering at Politecnico di Milano, Milano, Italy 2016. Currently, he is a PhD student in Electrical Engineering at Politecnico di Milano, Italy. His interests are in power electronics and electrical machines.

Giovanni Maria Foglia received his MS degree and the $\mathrm{PhD}$ in electrical engineering at Politecnico di Milano, Milano, Italy, in 1997 and 2000. Currently, he is an Assistant Professor at the Department of Energy at Politecnico di Milano, and his main field of interest is the analysis and design of PM electrical machines.

Matteo Felice Iacchetti (M'10) received the $\mathrm{PhD}$ in electrical engineering from the Politecnico di Milano, Milano, in 2008. Currently, he is a Lecturer with the School of Electrical and Electronic Engineering at The University of Manchester, Manchester, UK. His main research interests are the design, modeling and control of electrical machines.

Roberto Perini (M'10) received his MS degree and the $\mathrm{PhD}$ in electrical engineering from the Politecnico di Milano, Milano, Italy.

Currently, he is an Associate Professor at the Department of Energy at Politecnico di Milano. His interests are in the design and modeling of electrical machines and power electronics. 